\title{
Effect of thermal conductivity on Mhd heat and mass transfer: flow past an infinite vertical plate with Soret and Dufour effects
}

\author{
Halima Usman, Ime Jimmy Uwanta \\ Department of Mathematics, Usmanu Danfodiyo University, Sokoto, Nigeria \\ Email address: \\ hhalimausman@yahoo.com(H. Usman),imeuwanta@yahoo.com(I. J. Uwanta)
}

\section{To cite this article:}

Halima Usman, Ime Jimmy Uwanta. Effect of Thermal Conductivity on Mhd Heat and Mass Transfer: Flow Past an Infinite Vertical Plate with Soret and Dufour Effects. American Journal of Applied Mathematics. Vol. 1, No. 3, 2013, pp. 28-38.

doi: 10.11648/j.ajam.20130103.11

\begin{abstract}
This paper investigates the study of the effect of thermal conductivity on MHD flow past an infinite vertical plate with Soret and Dufour effects. The governing non-linear Partial Differential Equations are solved numerically by implicit finite difference scheme of Crank-Nicolson. Computations are performed for a wide range of the governing flow parameters such as thermal Grashof number, solutal Grashof number, magnetic field parameter, Prandtl number, Dufour number, Soret number, thermal conductivity and Schmidt number. The effects of these flow parameters on the velocity, temperature and concentration are shown graphically. It is observed that the velocity increases with the increase in Gr, Gc, $\mathrm{Du}, \mathrm{Ec}, \mathrm{k}$ and $\lambda$ and it decreases with the increase in $\mathrm{M}, \mathrm{Sr}$ and $\mathrm{b}$. Temperature decreases with an increase in $\gamma$ and Pr and concentration decreases with the increase in $\mathrm{Sr}$ and $\gamma$. Finally, the effects of various parameters on the skin friction coefficient, Nusselt number and Sherwood number are shown on Tables.
\end{abstract}

Keywords: Thermal Conductivity, MHD, Soret and Dufour Effects

\section{Introduction}

Combined heat and mass transfer by free convection in a porous medium has attracted considerable attention in the last decades, due to it is many important engineering and geophysical applications. Such flows have attracted the attention of a number of scholars due to their applications in many branches of science and technology, such as, in the field of agriculture engineering to study the underground water resources, seepage of water in river-beds, in petroleum technology to study the movement of natural gas, oil and water through oil reservoirs, in chemical engineering for filtration and purification processes, thermal and insulating engineering, modeling of packed sphere beds, solar power collector, pollutant dispersion in aquifers, cooling of electronic systems, ventilation of rooms, crystal growth in liquids, chemical catalytic reactors, grain storage devices, petroleum reservoirs, ground hydrology, fiber and granular insulation, nuclear waste repositories, high-performance building insulation, postaccident heat removal from pebble-bed nuclear reactors, concepts of aerodynamics heat shielding with transpiration cooling, etc. Bejan et al. [11], Ingham et al. [16] and Vafai [24]. The convection problem in porous medium has other applications in geothermal reservoirs and geothermal energy extractions. A comprehensive review of the studies of convective heat transfer mechanism through porous media has been made by Nield and Bejan, [19]. In view of the above mentioned effects, Alam and Rahman, [6] have studied Soret and Dufour effects on mixed convection flow past a porous flat plate with variable suction. Kafousiasis and Williaims [17] examined Soret and Dufour effects on mixed free-forced conductive and mass transfer boundary layer flow with temperature dependent viscosity. Anghel et al [9] investigated the Dufour and Soret effects on free convection boundary layer over a vertical surface embedded in a porous medium. Postelnica [23] studied numerically the influence of a magnetic field on heat and mass transfer by natural convection from vertical surfaces in porous media considering Soret and Dufour effects. Ajali and Uma [5] investigated thermal radiation effect over an electrically -conducting, Newtonian fluid in a steady laminar magneto hydrodynamic convective flow over a porous rotating infinite disk with the consideration of heat and mass transfer in the presence of Soret and Dufour 
numbers. Olarenwaju and Gbadeyan [21] examined the effects of Soret, Dufour, chemical reaction, thermal radiation and volumetric heat absorption/generation on mixed convection stagnation point flow on an isothermal vertical plate in a porous media. Gbadeyan et al. [14] investigated thermal diffusion and diffusion-thermo effects on combined heat and mass transfer on mixed convection boundary layer flow over a stretching vertical surface in a porous medium filled with a visco elastic fluid in the presence of magnetic field. Emmanuel et al. [13] studied numerically the effects of thermal diffusion and diffusionthermo on combined heat and mass transfer of a steady hydromagnetic convective and slip flow due to a rotating disk with viscous dissipation and ohmic heating. Anwar et al. [10] examined the combined effects of Soret and Dufour diffusion and porous impedance on laminar MHD mixed convection heat and mass transfer of an electricallyconducting, Newtonian, Boussinesq fluid from a vertical stretching surface in a Darcian porous medium under uniform transverse magnetic field. Abdulraheem et al. [1] have investigated a numerical solution for double diffusive free convective flow over a vertical stretching surface embedded in a porous medium in the presence of a homogeneous first order chemical reaction, radiation, Soret and Dufour effects. Bhupendra et al. [12] studied Soret and Dufour effects on unsteady MHD mixed convection flow past an infinite radiative vertical porous plate embedded in a porous medium in the presence of chemical reaction. Alam et al. [7] examined a steady two-dimensional free convection and mass transfer flow past a continuously moving semi-infinite vertical porous plate in a porous medium by taking into account the Dufour and Soret effects. Adrian [2] investigated heat and mass transfer characteristics of natural convection about a vertical surface embedded in a saturated porous medium subjected to a chemical reaction by taking into account the diffusionthermo (Dufour) and thermal-diffusion (Soret) effects. Very recently, Afif [3] presented an analysis of the non-Darcy MHD free convective heat and mass transfer past a vertical isothermal surface by including the analysis various effects such as Soret and Dufour effects, thermal dissipation and temperature-dependent viscosity. Olanrewaju [22] studied Dufour and Soret effects of a transient free convective flow with radiative heat transfer past a flat plate moving through a binary mixture. Afify [4] studied similarity solution in MHD: effects of thermal diffusion and diffusion-thermo on free convective heat and mass transfer over a stretching surface considering suction or injection. More recently Hayat et al. [15] investigated heat and mass transfer for Soret and Dufour's effect on mixed convection boundary layer flow over a stretching vertical surface in a porous medium filled with a visco elastic fluid.

Combined heat and mass transfer problems with Soret and Dufour effects are of importance in many processes and have received considerable focus in recent years. In processes such as drying, evaporation at the surface of water body, energy transfer in wet cooling tower and the flow in a desert cooler, heat and mass transfer occur simultaneously, Vafai [25]. In view of the above studies, the present work tends to study the effect of thermal conductivity on MHD heat and mass transfer flow past an infinite vertical plate taking into account the Dufour and Soret effects.

\section{Mathematical Formulation}

An unsteady two-dimensional flow of an incompressible and electrically conducting viscous fluid, along an infinite vertical porous flat plate embedded in a porous medium is considered. The x-axis is taken on the infinite plate, and parallel to the free-stream velocity which is vertical and the $\mathrm{y}$-axis is taken normal to the plate. A magnetic field $B_{0}$ of uniform strength is applied transversely to the direction of the flow. Initially the plate and the fluid are at same temperature $T_{\infty}$ in a stationary condition with concentration level $C_{\infty}$ at all points. For $\mathrm{t}>0$, the plate starts moving impulsively in its own plane with a velocity $U_{0}$, its temperature is raised to $T_{w}$ and the concentration level at the plate is raised to $C_{w}$. The fluid is assumed to have constant properties except that the influence of the density variations with temperature and concentration, which are considered only in the body force term. Under the above assumptions, the physical variables are functions of $y$ and $t$ only. Assuming that the Boussinesq and boundary-layer approximation hold and using the Darcy-Forchheimer model, the equations governing the problem are (see Alam and Rahman [8]):

$$
\begin{gathered}
\frac{\partial v^{*}}{\partial y^{*}}=0 \\
\frac{\partial u^{*}}{\partial t^{*}}+v \frac{\partial u^{*}}{\partial y^{*}}=v \frac{\partial^{2} u^{*}}{\partial y^{* 2}}-\frac{\sigma B_{0}{ }^{2} u^{*}}{\rho}-\frac{v u^{*}}{k^{*}}+ \\
g \beta\left(T^{*}-T_{\infty}^{*}\right)+g \beta^{*}\left(C^{*}-C_{\infty}^{*}\right) \\
\frac{\partial c^{*}}{\partial t^{*}}+v \frac{\partial c^{*}}{\partial y^{*}}=D \frac{\partial^{2} c^{*}}{\partial y^{* 2}}+D_{T} \frac{\partial^{2} T}{\partial y^{* 2}} \\
\frac{\partial T^{*}}{\partial t^{*}}+v \frac{\partial T^{*}}{\partial y^{*}}=\frac{1}{\rho C_{p}} \frac{\partial}{\partial y^{*}}\left[K(T) \frac{\partial T^{*}}{\partial y^{*}}\right]+ \\
\frac{D_{M}}{\rho C_{p}} \frac{\partial^{2} C^{*}}{\partial y^{* 2}}-b^{*} u^{2}+\frac{1}{\rho C_{p}}\left(\frac{\partial u^{*}}{\partial y^{*}}\right)^{2} \\
K(T)=k_{0}\left[1+\gamma\left(T-T_{\infty}\right)\right]
\end{gathered}
$$

The corresponding initial and boundary conditions are prescribed as follows: 


$$
\begin{array}{ll}
t \leq 0, & u^{\prime}=0, T^{\prime} \rightarrow T_{\infty}^{\prime}, \\
& C^{\prime} \rightarrow C_{\infty}^{\prime} \text { for all } y^{\prime} . \\
t>0, & u^{\prime}=u_{0}, T^{\prime} \rightarrow T_{w}^{\prime}, \\
& C^{\prime} \rightarrow C_{w}^{\prime} \text { at } y^{\prime}=0 . \\
& u^{\prime} \rightarrow 0, T^{\prime} \rightarrow T_{\infty}^{\prime}, \\
& C^{\prime} \rightarrow C_{\infty}^{\prime} \text { as } y \rightarrow \infty .
\end{array}
$$

Here $u^{*}$ and $v^{*}$ are the velocity components along and normal to the plate, $\boldsymbol{V}$ is the kinematic viscosity, $g$ is the acceleration due to gravity, $\beta$ is the coefficient of volume expansion, $\rho$ the density, $\beta^{*}$ is the volumetric coefficient of expansion with concentration, $\mathrm{K}(\mathrm{T})$ is the thermal conductivity, $C_{p}$ is the specific heat capacity at constant pressure, $k^{*}$ is the permeability of the porous medium, $D_{M}$ is the coefficient of mass diffusivity, $k_{0}$ is the thermal conductivity of the ambient fluid, D is the coefficient of diffusivity, $D_{T}$ is the coefficient of temperature diffusivity, $\gamma$ is the suction parameter, $\mathrm{b}$ is the joule heating parameter, $B_{0}$ is the magnetic field of constant strength. $T^{*}$ and $T_{\infty}^{*}$ are the temperature of the fluid inside the thermal boundary layer, $C^{*}$ and $C_{\infty}^{*}$ are the corresponding concentrations.

On introducing the following non-dimensional quantities:

$$
\begin{aligned}
& u=\frac{u^{*}}{u_{0}}, t=\frac{t^{*} u_{0}^{2}}{v}, y=\frac{u_{0} y^{*}}{v}, \theta=\frac{T^{*}-T_{\infty}^{*}}{T^{*}{ }_{w}-T_{\infty}^{*}}, \\
& C=\frac{C^{*}-C^{*} \infty}{C_{w}^{*}-C_{\infty}^{*}}, \operatorname{Pr}=\frac{v \rho C p}{u}, S c=\frac{v}{D}, \\
& b=\frac{b^{*} v}{u_{0}{ }^{2}\left(T^{*}-T_{\infty}^{*}\right)}, M=\frac{\sigma B_{0}{ }^{2} v}{\rho}, E c=\frac{u_{0}^{2}}{\rho C p\left(T^{*}{ }_{w}-T_{\infty}^{*}\right)} \text {, } \\
& G r=\frac{g \beta^{*}\left(T_{w^{*}}^{*}-T_{\infty}^{*}\right) v}{u_{0}^{3}}, G c=\frac{g \beta^{*}\left(C^{*}{ }_{w}-C_{\infty}^{*}\right) v}{u_{0}^{3}}, \\
& S r=v \frac{D_{T}\left(T^{*}{ }_{w}-T_{\infty}^{*}\right)}{v\left(C^{*}{ }_{w}-C_{\infty}^{*}\right)}, D u=\frac{D_{m}\left(C_{w}^{*}-C_{\infty}^{*}\right)}{v \rho C p\left(T^{*}{ }_{w}-T_{\infty}^{*}\right)}, \\
& \lambda=\gamma\left(T_{w}^{*}-T_{\infty}^{*}\right), \alpha=\frac{u_{0}}{v_{0}}
\end{aligned}
$$

Applying these non-dimensionless quantities (7), the set of equations (2), (3), (4), (5) and (6) reduces to the following:

$$
\begin{gathered}
\frac{\partial u}{\partial t}-\alpha \frac{\partial u}{\partial y}=\frac{\partial^{2} u}{\partial y^{2}}-M u- \\
\frac{1}{k} u+G r \theta+G c C \\
\frac{\partial C}{\partial t}-\alpha \frac{\partial C}{\partial y}=\frac{1}{S c} \frac{\partial^{2} C}{\partial y^{2}}+S r \frac{\partial^{2} \theta}{\partial y^{2}}
\end{gathered}
$$

$$
\begin{gathered}
\frac{\partial \theta}{\partial t}-\alpha \frac{\partial \theta}{\partial y}=\frac{\lambda}{\operatorname{Pr}\left(\frac{\partial \theta}{\partial y}\right)^{2}+\frac{1}{\operatorname{Pr}}(1+\lambda \theta) \frac{\partial^{2} \theta}{\partial y^{2}}+} \\
D u \frac{\partial^{2} C}{\partial y^{2}}-b u+E c\left(\frac{\partial u}{\partial y}\right)^{2}
\end{gathered}
$$

With the following initial and boundary conditions

$$
\begin{aligned}
& u=0, \theta=0, C=0, \quad \text { for all } y, \\
& u=1, \theta=1, C=1, \text { at } y=0, \\
& u=0, \theta=0, C=0 \text { as } y \rightarrow \infty .
\end{aligned}
$$

Where Ec is the Eckert number, Pr is the Prandtl number, $\mathrm{Sc}$ is the Schmidt number, $\mathrm{Sr}$ is the Soret number, $\mathrm{Du}$ is the Dufour number, $M$ is the Magnetic field parameter, $\mathrm{Gr}$ is the thermal Grashof number, Gc is the Solutal Grashof number, $\mathrm{b}$ is the joule heating parameter, $\lambda$ is the variable thermal conductivity, $\alpha$ is the variable suction parameter while $\mathrm{u}$ and $\mathrm{v}$ are dimensionless velocity components in $\mathrm{x}$ and $y$-dimensionless directions respectively and $t$ is the dimensionless time.

\section{Numerical Procedure}

To solve the unsteady coupled non-linear partial differential equations, a finite difference technique of implicit type namely Crank Nicolson implicit finite difference method which is always convergent and stable is employed. This method has been used to solve equations (8) - (10) subject to the boundary conditions (11). The partial differential equations are converted to difference equation. The Crank-Nicolson method has been used in several heat transfer, radiation and convection problems. The finite difference approximations to these equations are as follows:

$$
\begin{gathered}
\frac{u_{i}^{j+1}-u_{i}^{j}}{\Delta t}-\alpha\left(\frac{u^{j}{ }_{i+1}-u_{i}^{j}}{\Delta y}\right)= \\
\frac{u^{j}{ }_{i+1}+u^{j}{ }_{i-1}-2 u_{i}^{j}+u^{j+1}{ }_{i+1}+u^{j+1}{ }_{i-1}-2 u_{i}^{j+1}}{2(\Delta y)^{2}}- \\
M\left(\frac{u_{i}^{j+1}+u_{i}^{j}}{2}\right)-\frac{1}{K}\left(\frac{u_{i}^{j+1}+u_{i}^{j}}{2}\right)+ \\
G r\left(\frac{\theta_{i}^{j+1}+\theta_{i}^{j}}{2}\right)+G c\left(\frac{C_{i}^{j+1}+C_{i}^{j}}{2}\right) \\
\frac{C_{i}^{j+1}-C_{i}^{j}}{\Delta t}-\alpha\left(\frac{C^{j}{ }_{i+1}-C_{i}^{j}}{\Delta y}\right) \\
=\frac{1}{S c}\left(\frac{C^{j}{ }_{i+1}+C^{j}{ }_{i-1}-2 C_{i}^{j}+C^{j+1}{ }_{i+1}+C^{j+1}{ }_{i-1}-2 C_{i}^{j+1}}{2(\Delta y)^{2}}\right) \\
+S r\left(\frac{\theta_{i+1}^{j}+\theta^{j}{ }_{i-1}-2 \theta_{i}^{j}+\theta^{j+1}{ }_{i+1}+\theta^{j+1}{ }_{i-1}-2 \theta_{i}^{j+1}}{2(\Delta y)^{2}}\right)
\end{gathered}
$$




$$
\begin{aligned}
& \frac{\theta_{i}^{j+1}-\theta_{i}^{j}}{\Delta t}-\alpha\left(\frac{\theta^{j}{ }_{i+1}-\theta_{i}^{j}}{\Delta y}\right)= \\
& \frac{(1+\lambda \theta)}{\operatorname{Pr}}\left(\frac{\theta^{j}{ }_{i+1}+\theta^{j}{ }_{i-1}-2 \theta_{i}^{j}+\theta^{j+1}{ }_{i+1}+\theta^{j+1}{ }_{i-1}-2 \theta_{i}^{j+1}}{2(\Delta y)^{2}}\right)+ \\
& \frac{\lambda}{\operatorname{Pr}}\left(\frac{\theta^{j}{ }_{i+1}-\theta_{i}^{j}}{(\Delta y)^{2}}\right)+ \\
& D u\left(\frac{C^{j}{ }_{i+1}+C^{j}{ }_{i-1}-2 C_{i}^{j}+C^{j+1}{ }_{i+1}+C^{j+1}{ }_{i-1}-2 C_{i}^{j+1}}{2(\Delta y)^{2}}\right)- \\
& b\left(\frac{u_{i}^{j+1}+u_{i}^{j}}{2}\right)+E c\left(\frac{u_{i+1}^{j}-u_{i}^{j}}{\Delta y}\right)^{2}
\end{aligned}
$$

The initial and boundary conditions take the following forms:

$$
\begin{aligned}
& u_{i, j}=0, \quad \theta_{i, j}=0, \quad C_{i, j}=0, \text { for all } i \text { except } i=0, \\
& u_{0, j}=1, \quad \theta_{0, j}=1, \quad C_{0, j}=1, \\
& u_{M, j}=0, \quad \theta_{M, j}=0, C_{M, j}=0,
\end{aligned}
$$

where $M$ corresponds to $\infty$.

Equations (12), (13) and (14) are written in the following form:

$$
\begin{gathered}
-D_{1} U^{j+1}{ }_{i-1}+D_{2} U_{i}{ }^{j+1}-D_{1} U^{j+1}{ }_{i+1}= \\
D_{1} U^{j}{ }_{i-1}+D_{3} U_{i}^{j}+D_{4} U^{j}{ }_{i+1}+ \\
D_{5}\left(\theta_{i}^{j+1}+\theta_{i}^{j}\right)+D_{6}\left(C_{i}^{j+1}+C_{i}^{j}\right) \\
-E_{1} C^{j+1}{ }_{i-1}+E_{2} C_{i}^{j+1}-E_{1} C^{j+1}{ }_{i+1}= \\
E_{1} C^{j}{ }_{i-1}+E_{3} C_{i}^{j}+E_{4} C^{j}{ }_{i+1}+ \\
E_{5}\left(\theta^{j}{ }_{i+1}+\theta^{j}{ }_{i-1}-2 \theta_{i}^{j}+\theta^{j+1}{ }_{i+1}+\theta^{j+1}{ }_{i-1}-2 \theta_{i}^{j+1}\right) \\
-F_{1} \theta^{j+1}{ }_{i-1}+F_{2} \theta_{i}^{j+1}-F_{1} \theta^{j+1}{ }_{i+1}= \\
F_{1} \theta^{j}{ }_{i-1}+F_{3} \theta_{i}^{j}+F_{4} \theta^{j}{ }_{i+1}+F_{5}\left(\theta^{j}{ }_{i+1}-\theta_{i}^{j}\right)^{2} \\
+F_{6}\left(u^{j}{ }_{i+1}-u_{i}{ }^{j}\right)^{2}-F_{7}\left(u_{i}^{j+1}+u_{i}^{j}\right) \\
+F_{8}\left(C^{j}{ }_{i+1}+C^{j}{ }_{i-1}-2 C_{i}^{j}+C^{j+1}{ }_{i+1}+C^{j+1}{ }_{i-1}-2 C_{i}^{j+1}\right)
\end{gathered}
$$

Where: $h_{1}=\frac{\Delta t}{\Delta y}, h_{2}=\frac{\Delta t}{(\Delta y)^{2}}, h_{3}=\Delta t, h_{4}=M+\frac{1}{K}$,

$D_{1}=h_{2}, D_{2}=2\left(1+h_{2}+h_{3} h_{4}\right), D_{3}=2-2 \alpha h_{1}-2 h_{2}-h_{3} h_{4}$,

$D_{4}=2 \alpha h_{1}+h_{2}, D_{5}=h_{3} G r, D_{6}=h_{3} G c$,

$E_{1}=h_{2}, E_{2}=2\left(S c+h_{2}\right), E_{3}=2\left(-h_{2}+S c-h_{1} \alpha S c\right)$,

$E_{4}=2 h_{1} \alpha S c+h_{2}, E_{5}=h_{2} S c S r$,

$F_{1}=h_{2} F, F_{2}=2\left(\operatorname{Pr}+h_{2} F\right), F_{3}=2\left(-h_{2} F+\operatorname{Pr}-h_{1} \alpha \operatorname{Pr}\right)$,

$F_{4}=h_{2} F+2 r_{1} \alpha \operatorname{Pr}, F_{5}=2 h_{2} \lambda, F_{6}=2 h_{2} \operatorname{Pr} E c$,

$F_{7}=h_{3} b \operatorname{Pr}, F_{8}=h_{2} D u \operatorname{Pr}$.

Here the suffix $i$ refers to the space and $j$ refers to the time. $\Delta y$ and $\Delta t$ are the mesh sizes along $\mathrm{y}-$ direction and time $\mathrm{t}$ - direction respectively. Consequently, $\Delta t=t_{j+1}-t_{j}$ and $\Delta y=y_{i+1}-y_{i}$.

The skin friction, Nusselt number and Sherwood number are important physical parameters for this type of boundary layer flow. From the velocity, the skin friction is given by

$$
\tau^{\prime}=-\left.\mu \frac{\partial u^{\prime}}{\partial y}\right|_{y=0}
$$

and in view of equation (7), equation (16) reduces to

$$
\tau=-\left.\frac{\partial u}{\partial y}\right|_{y=0}
$$

where $\tau=\frac{\tau^{\prime}}{\rho u_{0}^{2}}$.

The rate of heat transfer coefficient, in terms of the Nusselt number is given by

$$
q=-\left.k \frac{\partial T^{\prime}}{\partial y^{\prime}}\right|_{y=0}
$$

In view of equation (7), equation (18) reduces to

$$
N u=\frac{q L}{k \Delta t}=-\left.\frac{\partial \theta}{\partial y}\right|_{y=o} .
$$

The rate of mass transfer coefficient, which in the dimensional form in terms of the Sherwood number, is given by

$$
\begin{gathered}
S h=-x \frac{\left(\frac{\partial C^{\prime}}{\partial y^{\prime}}\right)_{y=0}}{C_{w}^{\prime}-C_{\infty}^{\prime}} \Rightarrow \\
S h \operatorname{Re}_{x}^{-1}=-\left(\frac{\partial C}{\partial y}\right) \|_{y=0}
\end{gathered}
$$

where $\operatorname{Re}_{x}=\frac{V_{0} x}{v}$ is the Local Reynolds number.

\section{Numerical Solution}

Knowing the values of $\mathrm{u}, \mathrm{T}$ and $\mathrm{C}$ at a time $\mathrm{t}$, we can calculate the values at a time $t+\Delta t$ as follows. We substitute $i=2,3,4, \ldots, M-1$ in equation (12) which results in a tri-diagonal system of equations in unknown values of $u$. Using initial and boundary conditions, the system can be solved by Gaussian elimination method (Carnahan et al (18)). Thus $\mathrm{u}$ is known at all values of $y$ at time $t+\Delta t, y$ is chosen as $y_{\text {min }}=0$ and $y_{\max }=3.5$. Then knowing the values of $u$ and applying the same procedure and using boundary conditions, we can calculate $\mathrm{C}$ and $\mathrm{T}$ from equations (13) and (14) respectively. This procedure is continued to obtain the solution till desired 
time t. Computations are carried out for water vapour $(\mathrm{Pr}=$ $0.6)$, for air $(\operatorname{Pr}=0.71)$, for salt water $(\operatorname{Pr}=1.0)$, for hydrogen $(\mathrm{Sc}=0.22)$ and methanol $(\mathrm{Sc}=1.0)$.

\section{Results and Discussion}

\subsection{Velocity Profiles}

The effects of various thermo physical parameters on the fluid velocity are illustrated in figures 1 to 11 . Figures 1 and 2 depict the effect of the solutal and thermal Grashof's numbers Gc and Gr respectively. The velocity profile increases with increase in the values of $\mathrm{Gr}$ and $\mathrm{Gc}$ respectively. It is clearly seen in figure 6 that the effect of increasing the magnetic field strength on the momentum boundary layer thickness is illustrated. Increasing this parameter leads to a decrease in the velocity which confirmed the fact that the magnetic field results in a damping effect on the velocity by creating a drag force that opposes the field motion. Figure 5 illustrate the effect of variable thermal conductivity $\lambda(A)$ on the velocity profile. It is clearly seen that $\lambda>0$ corresponds to assisting flow (heated plate). Increase in the mixed convection parameter leads to an increase in the fluid velocity. Figure 4 displays the velocity profiles across the boundary layer from different values of dimensionless viscoelastic parameter $\mathrm{K}$ and it is observed that increasing $\mathrm{K}$ leads to an increase in the fluid velocity and thickening the velocity boundary layer. In figure 8 it is clearly seen that an increase in the value of the Schmidt number leads to a decrease in the velocity profile. This means that the larger the value of Sc, the thinner the momentum boundary layer size, hence decrease in the velocity.

The Prandtl number Pr, Soret number $\mathrm{Sr}$ and Suction parameter $\gamma(V)$ has little or no influence on the fluid velocity (see figures 10, 9 and 7). It is also interesting to note that increase in Dufour number Du and Eckert number Ec increases the fluid velocity (see figures 11 and 3).

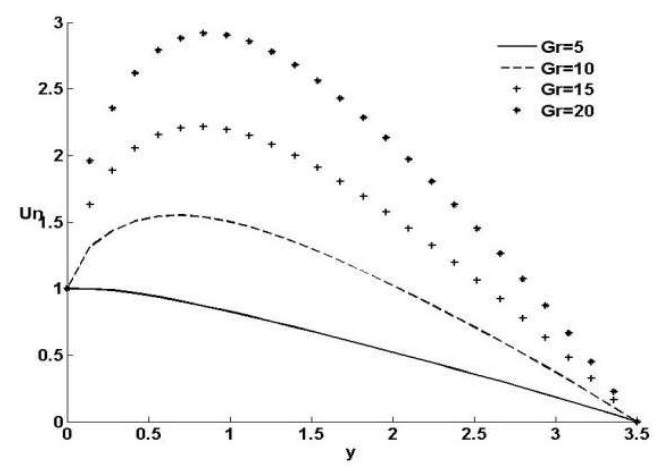

Fig.1: Velocity profiles for $V=0.1, \operatorname{Pr}=0.71, G c=5, A=3, S c=$ $0.22, E c=0.1, b=0.01, S r=0.2, K=1, M=1, D u=0.1$.

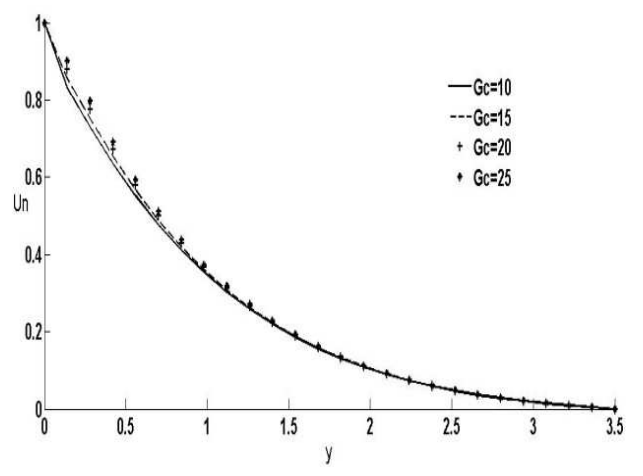

Fig. 2: Velocity profiles for $V=0.1, P r=0.71, G r=5, A=3, S c=$ $0.22, E c=0.1, b=0.01, S r=0.2, K=1, M=1, D u=0.1$.

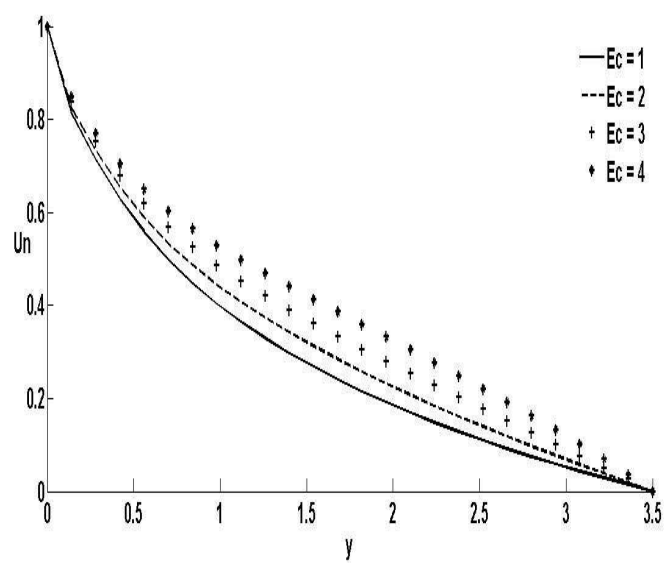

Fig. 3: Velocity profiles for $V=0.1, \operatorname{Pr}=0.71, G r=5, A=3, S c=$ $0.22, G c=5, b=0.01, S r=0.2, K=1, M=1, D u=0.1$.

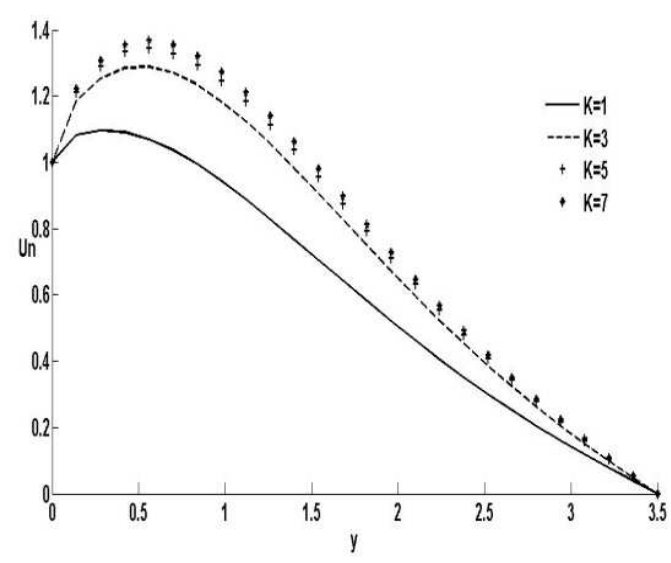

Fig. 4: Velocity profiles for $V=0.1, P r=0.71, G r=5, G c=5, A=3$, $S c=0.22, E c=0.1, S r=0.2, b=0.01, M=1, D u=0.1$. 


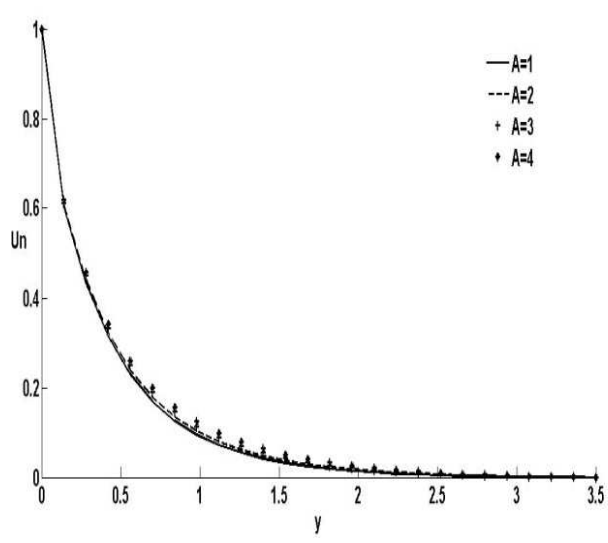

Fig. 5: Velocity profiles for $V=0.1, \operatorname{Pr}=0.71, G r=5, K=1, S c=0.22$, $E c=0.1, b=0.01, S r=0.2, M=1, D u=0.1, G c=5$.

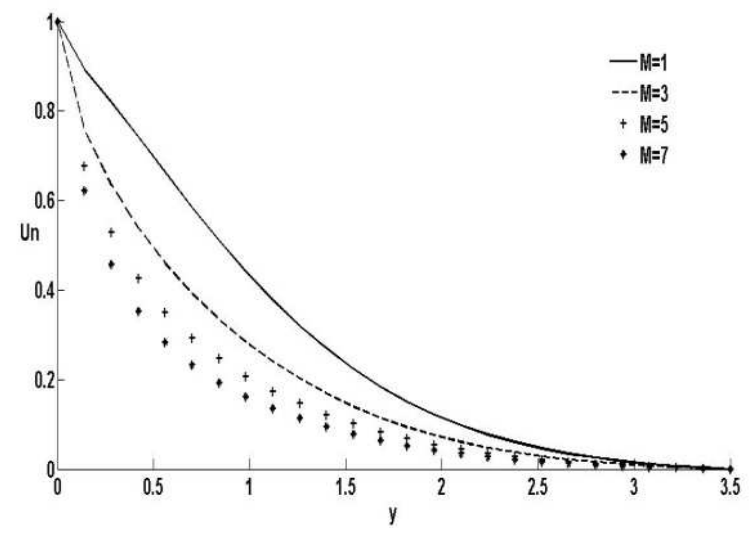

Fig. 6: Velocity profiles for $V=0.1, P r=0.71, G r=5, A=3, S c=0.22$, $E c=0.1, b=0.01, S r=0.2, K=1, G c=5, D u=0.1$.

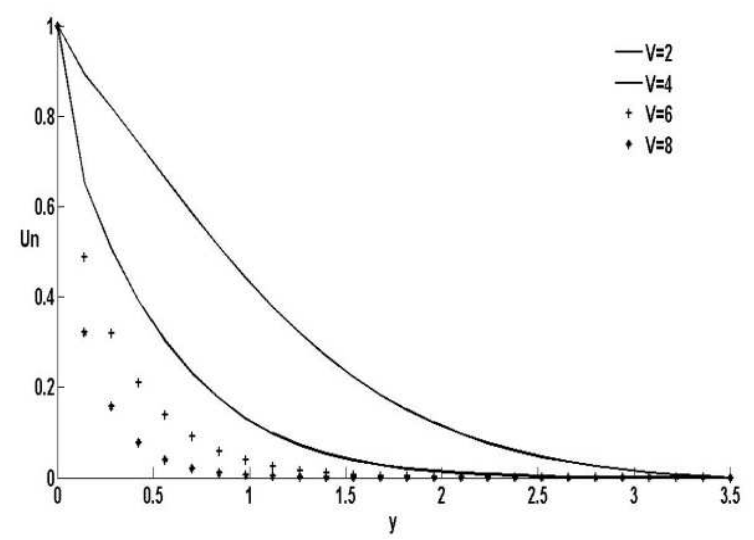

Fig. 7: Velocity profiles for $P r=0.71, G r=5, A=3, S c=0.22, E c=0.1$, $b=0.01, S r=0.2, K=1, M=1, D u=0.1, G c=5$.

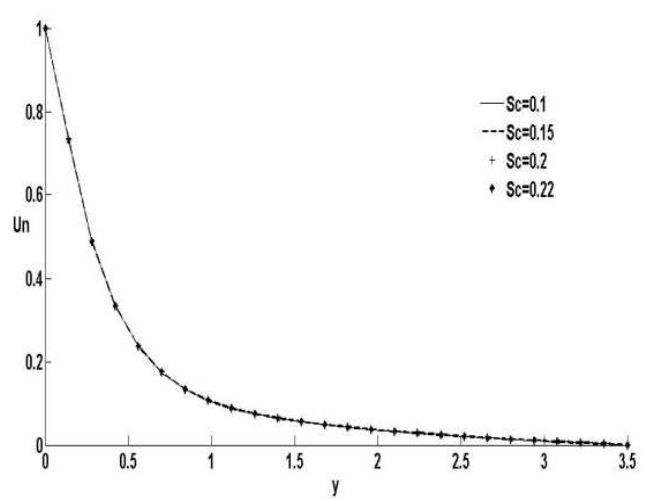

Fig. 8: Velocity profiles for $V=0.1, P r=0.71, G r=5, A=3, E c=0.1$, $b=0.01, S r=0.2, K=1, M=1, D u=0.1, G c=5$.

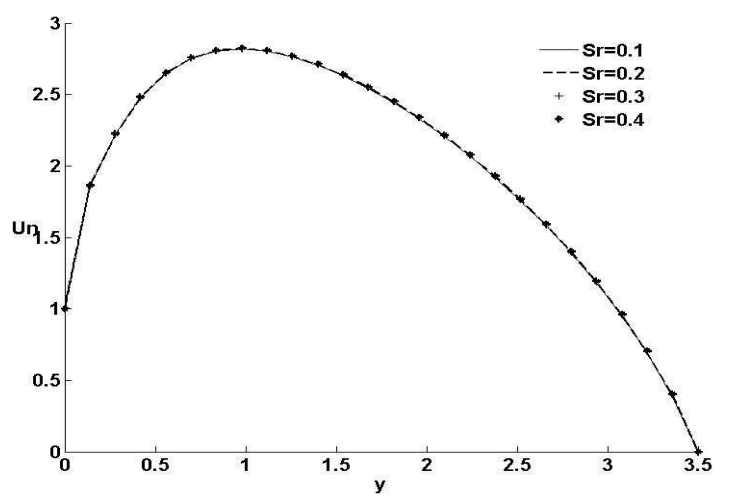

Fig. 9: Velocity profiles for $V=0.1, P r=0.71, G r=5, A=3, S c=0.22$, $E c=0.1, b=0.01, K=1, M=1, D u=0.1, G c=5$.

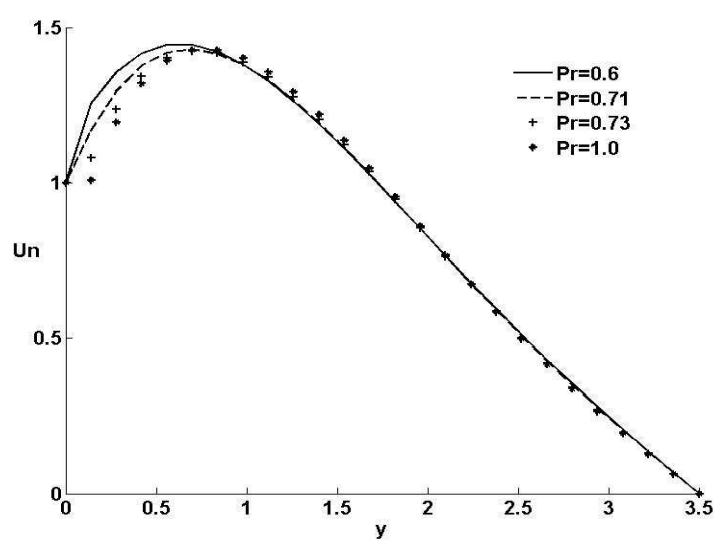

Fig. 10: Velocity profiles for $V=0.1, G r=5, A=3, S c=0.22, E c=0.1$, $b=0.01, S r=0.2, K=1, M=1, D u=0.1, G c=5$. 


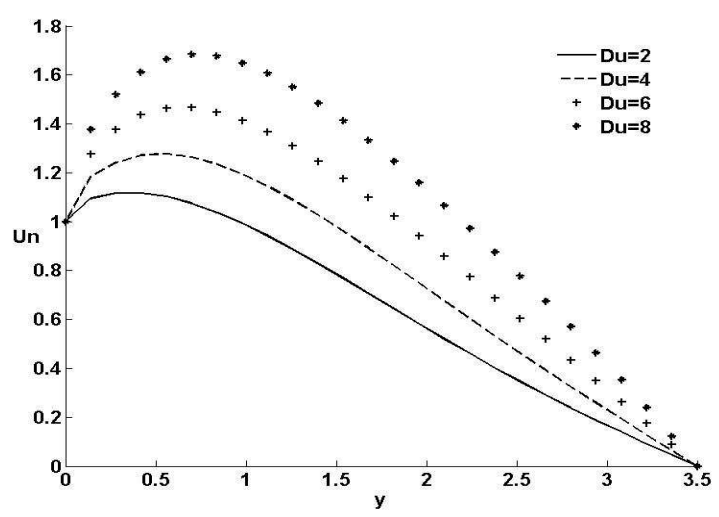

Fig. 11: Velocity profiles for $V=0.1, P r=0.71, G r=5, A=3, S c=0.22$, $E c=0.1, b=0.01, S r=0.2, K=1, M=1, G c=5.5 .2$. Temperature Profiles

The influences of various embedded parameters on the fluid temperature are illustrated in figures 12 to 17 . Figure 12 gives the variations of variable thermal conductivity $\lambda$ on the temperature. Increasing $\lambda$ leads to an increase in the temperature. Figure 13 shows the solution of temperature profiles across the boundary layer thickeness for different values of suction parameter $\gamma$. Increase in this parameter leads to a decrease in the temperature profile. It is clearly seen in figure 14 the effect of Prandtl number Pr on the temperature. The thermal boundary layer thikceness is found to increase upon increasing the prandtl number. However, figure 15 and 17 gives the effects of Dufour number Du and Eckert number Ec on temperature profiles. Increasing these parameters leads to an increase in the thermal boundary layer thickeness. Similarly, in figure 16, increase in Joule heating parameter $b$ decreases the thermal boundary layer.

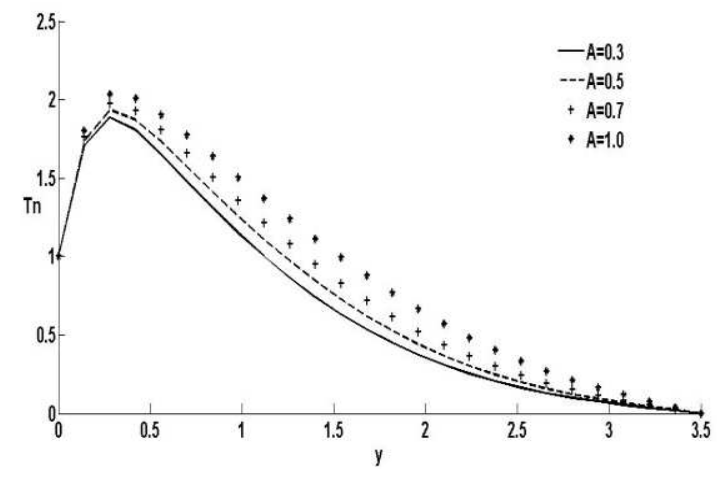

Fig. 12: Temperature profiles for $V=0.1, G r=5, G c=5, D u=2, S c=$ $0.22, E c=2, K=3, M=1, b=1, \operatorname{Pr}=0.71, S r=0.2$.

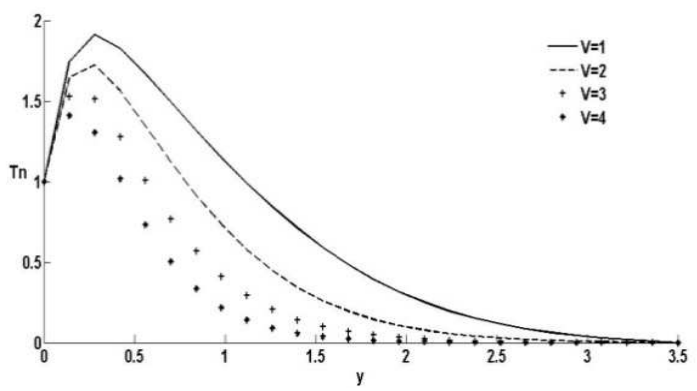

Fig. 13: Temperature profiles for $G r=5, G c=5, D u=2, S c=0.22, E c$ $=2, K=3, M=1, b=1, \operatorname{Pr}=0.71, S r=0.2, A=1$.

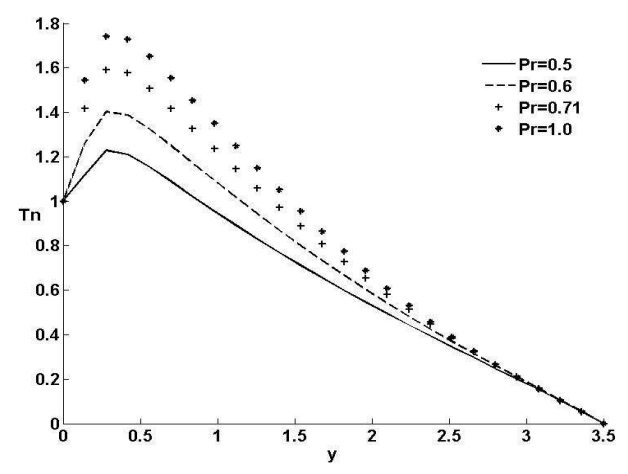

Fig. 14: Temperature profiles for $V=0.1, G r=5, G c=5, D u=2, S c=$ $0.22, E c=2, K=3, M=1, b=1, S r=0.2, A=1$.

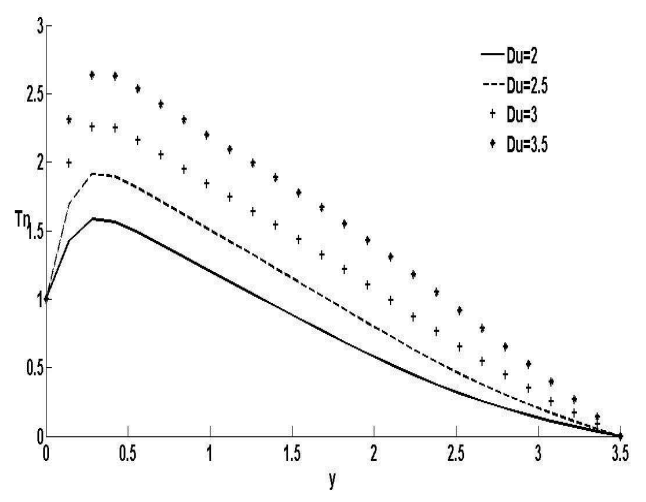

Fig. 15: Temperature profiles for $V=0.1, G r=5, G c=5, S c=0.22, E c$ $=2, K=3, M=1, b=1, P r=0.71, S r=0.2, A=1$.

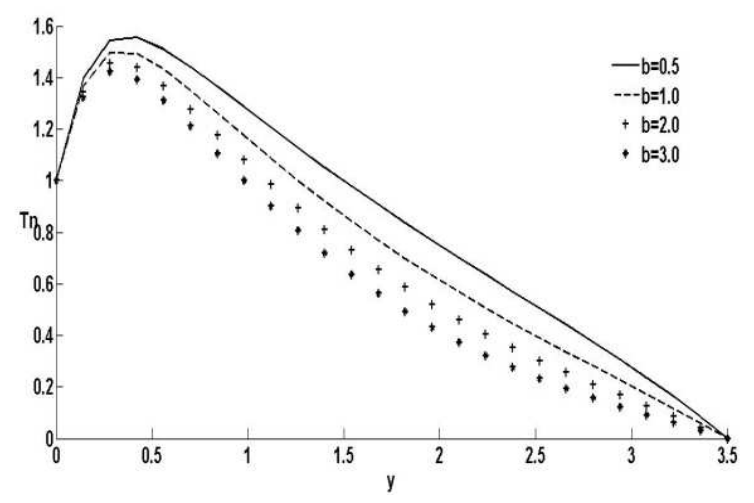

Fig. 16: Temperature profiles for $V=0.1, G r=5, G c=5, S c=0.22, E c$ =2, $K=3, M=1, \operatorname{Pr}=0.71, S r=0.2, A=1, D u=2$. 


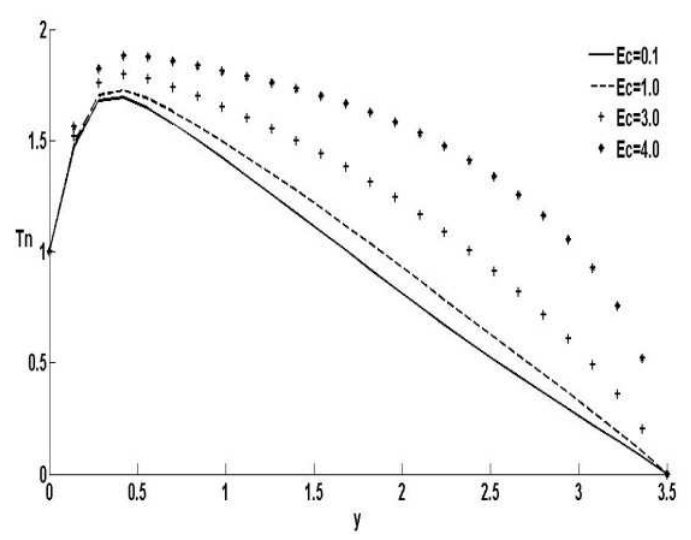

Fig. 17: Temperature profiles for $V=0.1, G r=5, G c=5, D u=2, S c=$ 0.22, $K=3, M=1, b=1, \operatorname{Pr}=0.71, S r=0.2, A=1.5 .3$. Concentration Profiles

The influences of various embedded thermophysical parameters on the fluid concentration had been illustrated in figures 18 to 21 . Figure 18 reflects that with increase in Sc the fluid concentratiom increases. Figure 19 is the graph of concentration profile against spanwise co-ordinate y for diifferent values of Soret numbers. It is note worthy that increase in this parameter decreases the concentration bounary layer thickenes. It is clearly seen that for a destructive suction parameter $\gamma$ the concentration boundary layer thickeness decreases ( see figure 21 ). The order of the reaction $\mathrm{Gr}$ has little effects on the concentration boundary layer thickeness. Increase in $\mathrm{Gr}$ leads to small decrease in the cocentration profile across the boundary layer ( see figure 20).

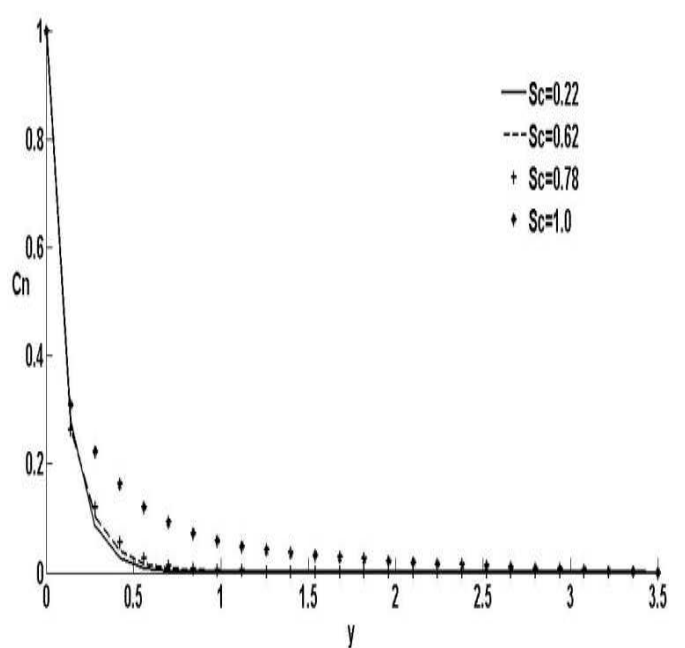

Fig. 18: Concentration profiles for $V=0.2, G r=5, G c=5, E c=1, b=$ $0.1, K=3, M=1, D u=3, S r=0.1, A=1, \operatorname{Pr}=0.71$.

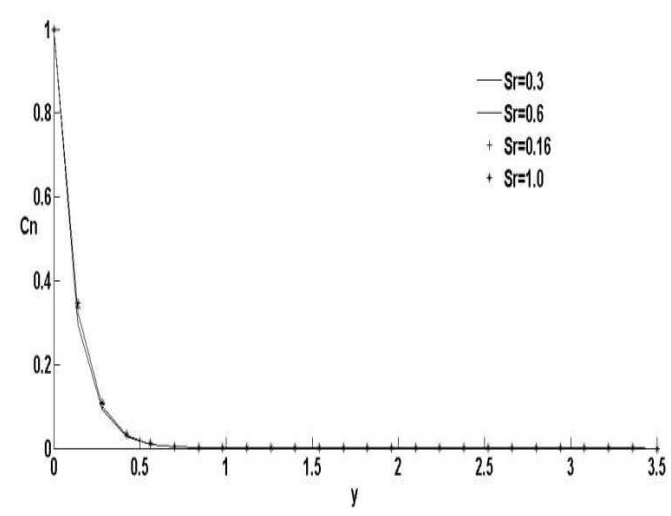

Fig. 19: Concentration profiles for $V=0.2, G r=5, G c=5, E c=1, b=$ $0.1, K=3, M=1, D u=3, S c=0.22, A=1, \operatorname{Pr}=0.71$.

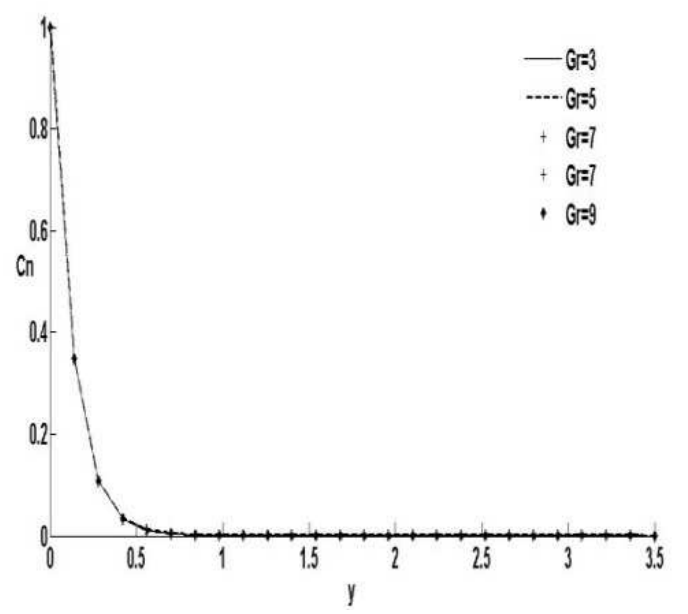

Fig. 20: Concentration profiles for $V=0.2, S c=0.22, G c=5, E c=1$, $b=0.1, K=3, M=1, D u=3, S r=0.1, A=1, \operatorname{Pr}=0.71$.

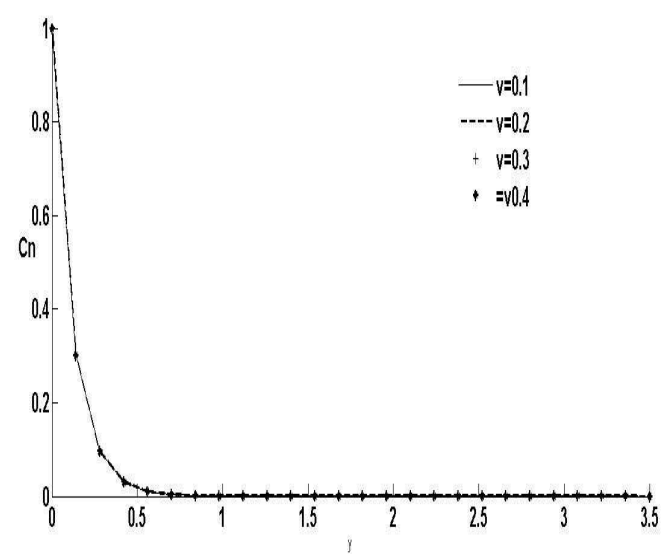

Fig. 21: Concentration profiles for $G r=5, G c=5, E c=1, b=0.1, K=$ 3, $M=1, D u=3, S r=0.1, A=1, \operatorname{Pr}=0.71, S c=0.22$.

The effects of various governing parameters on the skin friction, Nusselt number and Sherwood number are shown in tables 1 to 3, it is observed that as Gr, Gc, Pr, K, Ec and $\mathrm{Du}$ increases, the skin friction coefficient increases, where as the skin friction coefficient decreases as $\mathrm{M}$ decreases. 
From table 2, it is noticed that the Nusselt number increases as $\mathrm{Pr}$ and Ec are increased, while as the joule heating parameter $b$ increases, the Nusselt number decreases. From table 3, it is found that as Sc, Sr and suction parameter increases, the Sherwood number decreases.

Table 1: Effect of $G r, G c, P r, M, K$, Ec and Du on Skin friction $(\tau)$

\begin{tabular}{llllllll}
\hline $\mathbf{G r}$ & $\mathbf{G c}$ & $\mathbf{P r}$ & $\mathbf{M}$ & $\mathbf{K}$ & $\mathbf{E c}$ & $\mathrm{Du}$ & $\tau$ \\
\hline 5 & 5 & 0.71 & 0.5 & 1.0 & 0.1 & 2.0 & 0.0638 \\
$\mathbf{1 0}$ & 5 & 0.71 & 0.5 & 1.0 & 0.1 & 2.0 & 0.0868 \\
5 & $\mathbf{1 0}$ & 0.71 & 0.5 & 1.0 & 0.1 & 2.0 & 0.0653 \\
5 & 5 & $\mathbf{1 . 0}$ & 0.5 & 1.0 & 0.1 & 2.0 & 0.1071 \\
5 & 5 & 0.71 & $\mathbf{1 . 0}$ & 1.0 & 0.1 & 2.0 & 0.0601 \\
5 & 5 & 0.71 & 0.5 & $\mathbf{2 . 0}$ & 0.1 & 2.0 & 0.0686 \\
5 & 5 & 0.71 & 0.5 & 1.0 & $\mathbf{0 . 2}$ & 2.0 & 0.0639 \\
5 & 5 & 0.71 & 0.5 & 1.0 & 0.1 & $\mathbf{3 . 0}$ & 0.0728 \\
\hline
\end{tabular}

$(\mathrm{A}=1, \mathrm{~V}=0.2, \mathrm{Sc}=0.22, \mathrm{Sr}=0.2, \mathrm{~b}=0.01)$

Table 2: Effect of $\mathrm{Gr}, \mathrm{Pr}, \mathrm{Du}, \mathrm{Ec}$ and $\mathrm{b}$ on Nusselt number (Nu).

\begin{tabular}{llllll}
\hline $\mathbf{P r}$ & Du & $\mathbf{b}$ & $\mathbf{E c}$ & $\mathbf{G r}$ & $\mathbf{N u}$ \\
\hline 0.71 & 2.0 & 0.01 & 0.1 & 5 & 0.0759 \\
0.71 & 2.0 & 0.01 & 0.1 & $\mathbf{1 0}$ & 0.0868 \\
0.71 & $\mathbf{3 . 0}$ & 0.01 & 0.1 & 5 & 0.0758 \\
0.71 & 2.0 & 0.01 & $\mathbf{0 . 2}$ & 5 & 0.1020 \\
$\mathbf{1 . 0}$ & 2.0 & 0.01 & 0.1 & 5 & 0.1071 \\
0.71 & 2.0 & $\mathbf{0 . 0 2}$ & 0.1 & 5 & 0.0587 \\
\hline
\end{tabular}

$(\mathrm{Gc}=5, \mathrm{~A}=1, \mathrm{M}=0.5, \mathrm{k}=1, \mathrm{Sr}=0.2, \mathrm{Sc}=0.22, \mathrm{~V}=0.2)$

Table 3: Effect of $G r, S c, S r, E c$ and V on Sherwood number (Sh).

\begin{tabular}{llllll}
\hline $\mathbf{G r}$ & $\mathbf{S c}$ & $\mathbf{S r}$ & $\mathbf{E c}$ & $\mathbf{V}$ & $\mathbf{S h}$ \\
\hline 5 & 0.22 & 0.2 & 0.1 & 0.2 & 0.0126 \\
$\mathbf{1 0}$ & 0.22 & 0.2 & 0.1 & 0.2 & 0.0117 \\
5 & $\mathbf{0 . 3}$ & 0.2 & 0.1 & 0.2 & 0.0124 \\
5 & 0.22 & $\mathbf{0 . 3}$ & 0.1 & 0.2 & 0.0115 \\
5 & 0.22 & 0.2 & $\mathbf{0 . 2}$ & 0.2 & 0.0117 \\
5 & 0.22 & 0.2 & 0.1 & $\mathbf{0 . 6}$ & 0.0116 \\
\hline
\end{tabular}

$(\mathrm{Pr},=0.71, \mathrm{Ec}=0.1, \mathrm{M}=0.5, \mathrm{~A}=1, \mathrm{~K}=1, \mathrm{Du}=2, \mathrm{~b}=0.01, \mathrm{Gc}=5)$

\section{Conclusions}

In this paper we have studied numerically the effect of thermal conductivity on MHD heat and mass transfer flow past an infinite vertical plate with Soret and Dufour effects. The non-dimensional governing equations are solved with the help of implicit finite difference method of CrankNicolson type. The conclusions of the study are as follows:

1. The velocity increases with the increase in thermal Grashof number and solutal Grashof number.

2. The velocity decreases with an increase in the magnetic parameter.

3. The velocity as well as the temperature increases with an increase in Dufour number, Eckert number and thermal conductivity.

4. Increasing the suction parameter reduces velocity, temperature and concentration.

5. The velocity as well as the concentration decreases with an increase in Soret number.

6. Increasing the Prandtl number substantially decreases the translational velocity and increases the temperature profile.

7. An increase in the Schmidt number leads to increase in the concentration.

8. Increasing the joule heating parameter decreases the translational velocity.

9. The velocity increases with an increase in the permeability of the porous medium parameter.

\section{Nomenclature}

$C$ - Concentration

$C_{p}$ - Specific heat at constant pressure

D - Mass diffusivity

$D_{M}$ - Coefficient of Mass diffusivity

$D_{T}$ - Coefficient of temperature diffusivity

$\mathrm{Du}$ - Dufour number

$g$-Acceleration due to gravity

$\mathrm{Gr}$ - Grashof number

Gc - Solutal Grashof number

$K$ - Permeability of the porous medium

$\mathrm{Nu}-$ Nusselt number

$\mathrm{Pr}-$ Prandtl number

Sc - Schmidt number

$\mathrm{Sr}-$ Soret number

$T$ - Temperature

$u, v$-velocities in the $x$ and $y$-direction respectively

$x, y$-Cartesian coordinates along the plate and normal to it, respectively

$\rho$ - Density of the fluid

$B_{o}$ - Magnetic field of constant strength

Ec-Eckert number

$\mathrm{M}$ - Magnetic field parameter

$\mathrm{b}$ - Joule heating parameter 


\section{Greek Letters}

$\beta^{*}$ - coefficient of expansion with concentration

$\beta$ - Coefficient of thermal expansion

$\mu$-coefficient of viscosity

$\rho$ - Density of fluid

$\theta$ - dimensionless temperature

$v$ - Kinematic viscosity

$\alpha$ - Chemical reaction parameter

$\gamma$ - Suction parameter

$\lambda$ - Variable thermal conductivity

\section{Subscripts}

$w$ - condition at wall

$\infty$ - condition at infinity

\section{References}

[1] Abdulraheem, M. A., Mansour, M. A. and Chamkha, A. J., (2011). Effects of Soret and Dufour numbers on free convection over Isothermal and Adiabatic stretching surfaces embedded in porous media. Journal of porous media, DOI: 10, 1615/ Journal Porous Media, 14(1), pp. 67 72.

[2] Adrian P., (2007). Influence of chemical reaction on heat and mass transfer by natural convection from vertical surfaces in porous media considering Soret and Dufour effects. Heat Mass Transfer, 43: pp. 595-602.

[3] Afif A., (2006). Effects of Thermal diffusion and diffusion Thermo on non-Darcy MHD free convective flow and mass transfer past a vertical isothermal isothermal surface embedded in a porous medium with thermal dispersion and temperature dependent viscosity. Applied Math Model.

[4] Afify, A.A., (2009). Similarity solution in MHD: Effects of thermal-diffusion and diffusion-thermo on free convective heat and mass transfer over a stretching surface considering suction or injection. Communication in Non-Linear Science Numerical Simulation, 14: pp. 2202-2214.

[5] Ajali, D. S. P., and Uma, D. R. (2010). Soret and Dufour effects on MHD slip flow with thermal radiation over a porous rotating infinite disk. Communications in Non Linear Science and Numerical Simulation, 16(4), pp. 19171930.

[6] Alam, M. S., and Rahman, M. M., (2006). Dufour and Soret effects on mixed convection flow past a vertical porous plate with variable porous with variable suction. Non-linear Analysis: Modeling and control, 11(10), pp. 3-12.

[7] Alam, M.S., Ferdows, M., Ota, M., and Maleque, M.A. (2006). Dufour and Soret effects on Steady Free Convection and Mass transfer Flow past a semi-infinite Vertical Porous Plate in a Porous Medium. International Journal of Applied Mechanics and Engineering, 11(3), pp.535-545.

[8] Alam, M. S. and Rahman, M. M. (1992). Dufour and Soret effects on MHD free convective and mass transfer flow past a vertical flat plate embedded in a porous medium. Journal of Naval Architecture and Marine Engineering. 2(1): pp. 369-374.
[9] Anghel, M., Takher, H. S. and Pop, I., (2000). Dufour and Soret effects on free convection boundary layer over a vertical surface embedded in a porous medium. Studia Universitatis Basas-BolyalMathematica, XLV (4), pp. 11-21.

[10] Anwar, B.O., Bakier, A.Y.,and Prasad V.R., (2009). Numerical study of free convection magneto hydrodynamic heat and mass transfer from a stretching surface to a saturated porous medium with Soret and Dufour effects. Computational Material Science 46, pp. 57-65.

[11] Bejan, A., Dincer, I., Lorente, S., Miguel, A. F. and Reis, A. H. (2004). Porous and Complex Flow Structures in Modern Technologies.Springer. New York, NY.

[12] Bhupendra, K. S., Kailash, Y.Nidhish, K., Mishra, R. C., and Chaudhary, R. C. (2012). Soret and Dufour Effects on Unsteady MHD Mixed Convection Flow past a Radiative Vertical Porous Plate Embedded in a Porous Medium with Chemical Reaction. Applied Mathematics, 3, pp. 717-723 doi: $10.4236 / \mathrm{am}$.

[13] Emmanuel, O, Jonathan, S, and Robert, H., (2011). Thermal-diffusion and diffusion-thermo effects on combined Heat and Mass transfer of a steady MHD hydro convective and slip flow due to a rotating disk with viscous dissipation and ohmic heating. Global Journal of Science Frontier Research, International Communications in Heat and Mass Transfer, 35: pp. 908-915.

[14] Gbadeyan, J. A., Idowu, A. S., Ogunsola, A. W., Agbola, O. O., and Olanrewaju, P. O. (2011). Heat and Mass transfer for Soret and Dufour's effect on mixed convection boundary layer flow over a stretching vertical surface in a porous medium filled with a visco elastic fluid in the presence of a magnetic field. Global Journal of Science Frontier Research. 11(8), pp. 97-114.

[15] Hayat, T., Mustafa, M. and Pop, I., (2010). Heat and Mass transfer for Soret and Dufour's effect on mixed convection boundary layer flow over a stretching vertical surface in a porous medium filled with a visco elastic fluid. Communications in Non Linear Science and Numerical Simulation. 15, pp.1183-1196.

[16] Ingham, D. B. and Pop, I., (1998). Transport phenomena in porous media. Vol. II. Pergamon: Oxford, UK.

[17] Kafousiasis, N. G., and Williams, E. M. (1995). Thermaldiffusion and diffusion-Thermo effects on mixed free-forced convective and Mass transfer boundary layer flow with temperature dependent Viscosity. International Journal of Engineering Science, 33, pp. 1369-1384.

[18] Carnahan, H., Luther, H. A., and Wilkes, J.O., (1969). Applied Numerical Methoda, John Willey, newYork.

[19] Nield, D. A. and Bejan, A., (1998). Convection in Porous media, Second edition, Springer, New York.

[20] Olarenwaju, P. O., and Gbadeyan, J.A. (2011). Effects of Soret, Dufour, and Chemical reaction, Thermal Radiation and Volumetric Heat Generation / Absorption on mixed convection stagnation point flow on an Isothermal Vertical plate in porous media. Pacific Journal of Science and Technology. 12(2): pp. 234-245.

[21] Olanrewaju, P. O., (2010). Dufour and Soret Effects of a Transient free convective flow with Radiative heat transfer past a flat plate moving through a binary mixture. Pacific Journal of Science and Technology, 11(1): pp. 673-685. 
[22] Postelnica, A. (2007). Influence of a magnetic field on Heat and Mass transfer by natural convection from vertical surfaces in porous media considering Soret and Dufour effects. International Journal of Heat Mass transfer, 43, pp. 595-602.
[23] Vafai, K. (2000). Hand book of porous media, second edition. Taylor and Francis: New York.

[24] Vafai, K. (2005). Hand book of porous media, second edition. Taylor and Francis: New York. 